\title{
Lattice Boltzmann simulations of spontaneous flow in active liquid crystals: the role of boundary conditions
}

\author{
D. Marenduzzo ${ }^{1}$, E. Orlandini' ${ }^{2}$, M. E. Cates ${ }^{1}$, J. M. Yeomans ${ }^{3}$ \\ ${ }^{1}$ SUPA, School of Physics, University of Edinburgh, \\ Mayfield Road, Edinburgh EH9 3JZ, Scotland \\ ${ }^{2}$ Dipartimento di Fisica and Sezione INFN, \\ Universita' di Padova, Via Marzolo 8, 35131 Padova, Italy \\ ${ }^{3}$ The Rudolf Peierls Centre for Theoretical Physics, \\ 1 Keble Road, Oxford OX1 3NP, England
}

\begin{abstract}
Active liquid crystals or active gels are soft materials which can be physically realised e.g. by preparing a solution of cytoskeletal filaments interacting with molecular motors. We study the hydrodynamics of an active liquid crystal in a slab-like geometry with various boundary conditions, by solving numerically its equations of motion via lattice Boltzmann simulations. In all cases we find that active liquid crystals can sustain spontaneous flow in steady state contrarily to their passive counterparts, and in agreement with recent theoretical predictions. We further find that conflicting anchoring conditions at the boundaries lead to spontaneous flow for any value of the 'activity' parameter, while with unfrustrated anchoring at all boundaries spontaneous flow only occurs when the activity exceeds a critical threshold. We finally discuss the dynamic pathway leading to steady state in a few selected cases.
\end{abstract}




\section{INTRODUCTION}

Active viscoelastic gels and active liquid crystals are a new kind of soft materials [1, 2, 3, 4, 5]. They differ from their passive counterparts in the crucial respect that they continuously burn energy, e.g. from chemical reactions, which drives them out of thermodynamic equilibrium even in steady state. Examples of passive viscoelastic fluids are polymeric fluids, rubber, molten plastics etc. Examples of active gels are cytoskeletal slabs [6, 7], biomimetic systems [8] or solutions of molecular motors such as dyneins or myosins and microtubules or actin fibers [6, 7, 8, 9, 10, 11]. In these solutions the molecular motors interact with actin or microtubules by exerting forces or torques on them, creating motile and transient cross-links etc. It is the presence of the molecular motors, which are able to use chemical energy to perform mechanical work, which renders these viscoelastic polymeric fluids 'active'. The source of chemical energy in such active gels typically comes from ATP hydrolysis (there is estimated to be $\sim 15 k_{B} T$ worth of biochemical energy from each ATP hydrolysis reaction). It has also been suggested that the equations of motion of a continuum active gel may explain some macroscopic aspects of motility in bacterial colonies, such as swarming (i.e. the collective behaviour of a large number of bacterial swimmers), elastotaxis etc. [3, 4].

In general, the modelling of active soft matter presents novel fundamental challenges to theoreticians, due to the non-existence of a thermodynamic free energy. Furthermore, the elastic and rheological properties of these materials may be anticipated to be strongly affected by their nonequilibrium nature ('activity'). E.g., we now know that spontaneous flow can exist in steady state in active materials [1, 2], in sharp contrast to what happens in their passive counterparts, where any non-zero current is disallowed in equilibrium by momentum conservation and the Navier-Stokes equations. Furthermore, many biological gels, such as actin and neurofilament networks, thicken when sheared [12], which is the opposite of the typical behaviour of viscous polymeric fluids, molten plastics etc., which flow more easily as the external forcing driving their flow increases.

Our goal here is to study numerically the equations of motion of an active liquid crystal sample confined between two parallel plates in a quasi-1D geometry, focussing on the effects of different anchoring conditions for the director field (the active analog of the director field), and of the fluid behaviour deep in the active phase. Numerical methods are needed in this, as the insight which is possible to obtain analytically is limited although important. For instance, it has been possible to theoretically predict [2] by analytic methods the existence of an activity-induced transition between a passive phase with no local flow to an 'active' phase, in which the fluid is flowing at steady state. However, as the techniques used thus far essentially allow a stability analysis of the problem in selected geometries, it is not possible to predict e.g. the flowing and director field patterns deep in the active phase, away from the transition to spontaneous flow. Furthermore, using a numerical method allows us to consider more detailed forms for the equations of motion. Thus here we extend the vector polarisation model considered analytically in Ref. [1, 2], but a tensorial equivalent (similar to the one introduced in Ref.[3, 4] ]), which allows us to study situations in which the order parameter is not constant throughout the sample (as occurs in passive liquid crystals e.g. 
in shear banding and close to a defect core).

We will show that the existence of elastic frustration in the sample rising from the anchoring at the boundaries is important in determining the steady state solution, just as it is in selecting director field patterns of a passive liquid crystal under an imposed shear. Here we focus on two examples of boundary conditions. With normal anchoring, we find a transition between passive and active phase at a (size-dependent) non-zero value of the 'activity' parameter. With conflicting anchoring (we choose the anchoring leading to the hybrid aligned nematic - HAN - cell in conventional passive liquid crystals), on the other hand, the behaviour is quite different and the threshold is zero, i.e. any active liquid crystal in a HAN geometry spontaneously flows in steady state. Increasing the activity (going deeper in the active phase), results in other 'transitions'. In the normal anchoring case we observe banded flow, with the number of bands increasing as we go deeper into the active phase, while in the HAN cell geometry a Poiseuille-like flow sets up, and changes direction upon increasing the activity, resulting in vastly different elastic deformations of the sample in steady state. We also follow the dynamic pathway leading to steady state in selected cases and find that transiently the director field may be transiently asymmetric although it is eventually symmetric in steady state. Furthermore we observed some dependence of the steady state we find in our simulations on the initial conditions, hence on the history of the system.

This work is structured as follows. In Section II we introduce the tensorial model we consider and the equations of motion we want to solve. We also briefly show how the lattice Boltzmann algorithm of Ref. [13] may be generalised to solve these equations of motion. In Section III we report our results for the flow and director field patterns in steady state in the case of normal (Section III A) and conflicting (Section III B) boundary conditions at the sample walls. In both cases we assume translational invariance parallel to the walls so that the numerical problem is effectively one dimensional. We also discuss the dynamics leading to steady state in a selected case with normal anchoring (Section III C). Finally, Section IV contains our conclusions.

\section{MODEL AND METHODS}

We introduce a Landau-de Gennes free energy to describe the equilibrium physics of the active liquid crystal. This is a function of a tensorial order parameter, $Q_{\alpha \beta}$, whose largest eigenvalue gives the strength of local order. The free energy $\mathcal{F}$ is a sum of two terms, $\mathcal{F}_{1}+\mathcal{F}_{2}$. The first one is a bulk contribution,

$$
\mathcal{F}_{1}=\frac{A_{0}}{2}\left(1-\frac{\gamma}{3}\right) Q_{\alpha \beta}^{2}-\frac{A_{0} \gamma}{3} Q_{\alpha \beta} Q_{\beta \gamma} Q_{\gamma \alpha}+\frac{A_{0} \gamma}{4}\left(Q_{\alpha \beta}^{2}\right)^{2}
$$

while the second, $\mathcal{F}_{2}=K\left(\partial_{\gamma} Q_{\alpha \beta}\right)^{2} / 2$ is a distortion term [15]. Hereafter Greek indices denote Cartesian components and summation over repeated indices is implied. $K$ is an elastic constant. We have taken the one elastic costant approximation and neglected the spontaneous splay coefficient, which is equivalent to having infinitely strong anchoring at the boundaries. Adding a spontaneous splay does not change the physics qualitatively as argued in [2]. In the free energy 
equation, $A_{0}$ is a constant and $\gamma$ controls the magnitude of order. The anchoring of the director field on the boundary surfaces is along $\hat{x}$ or $\hat{x}$, see Fig. 1.

The equation of motion for $\mathbf{Q}$ is then [16]

$$
\left(\partial_{t}+\vec{u} \cdot \nabla\right) \mathbf{Q}-\mathbf{S}(\mathbf{W}, \mathbf{Q})=\Gamma \mathbf{H}+\lambda \Delta \mu \mathbf{Q}
$$

where $\Gamma$ is a collective rotational diffusion constant, $\Delta \mu$ is the chemical potential gained via ATP hydrolysis [1], and $\lambda$ is an 'activity' parameter of the liquid crystal.

Eq. (2) is valid to first order in $\Delta \mu$ [1]. The first term on the left-hand side of Eq. (2) is the material derivative describing a quantity advected by a fluid with velocity $\vec{u}$. This is generalized for rod-like molecules by a second term

$$
\begin{aligned}
\mathbf{S}(\mathbf{W}, \mathbf{Q}) & =(\xi \mathbf{D}+\omega)(\mathbf{Q}+\mathbf{I} / 3) \\
& +(\mathbf{Q}+\mathbf{I} / 3)(\xi \mathbf{D}-\omega)-2 \xi(\mathbf{Q}+\mathbf{I} / 3) \operatorname{Tr}(\mathbf{Q W})
\end{aligned}
$$

where $\operatorname{Tr}$ denotes the tensorial trace, while $\mathbf{D}=\left(\mathbf{W}+\mathbf{W}^{T}\right) / 2$ and $\omega=\left(\mathbf{W}-\mathbf{W}^{T}\right) / 2$ are the symmetric part and the anti-symmetric part respectively of the velocity gradient tensor $W_{\alpha \beta}=$ $\partial_{\beta} u_{\alpha}$. The constant $\xi$ depends on the molecular details of a given liquid crystal. In our context increasing $\xi$ tends to disfavour tumbling and favour aligning of the director in the material. The molecular field $\mathbf{H}$ in Eq. 2 is given by $\mathbf{H}=-\frac{\delta \mathcal{F}}{\delta \mathbf{Q}}+(\mathbf{I} / 3) \operatorname{Tr} \frac{\delta \mathcal{F}}{\delta \mathbf{Q}}$.

The fluid velocity, $\vec{u}$, obeys the continuity equation and the Navier-Stokes equation,

$$
\begin{aligned}
\rho\left(\partial_{t}+u_{\beta} \partial_{\beta}\right) u_{\alpha} & =\partial_{\beta}\left(\Pi_{\alpha \beta}\right)+\eta \partial_{\beta}\left(\partial_{\alpha} u_{\beta}+\partial_{\beta} u_{\alpha}\right. \\
& \left.+\left(1-3 \partial_{\rho} P_{0}\right) \partial_{\gamma} u_{\gamma} \delta_{\alpha \beta}\right),
\end{aligned}
$$

where $\rho$ is the fluid density and $\eta$ is an isotropic viscosity, and $\Pi_{\alpha \beta}=\Pi_{\alpha \beta}^{\text {passive }}+\Pi_{\alpha \beta}^{\text {active }}$. $\Pi_{\alpha \beta}^{\text {passive }}$ is the stress tensor necessary to describe ordinary liquid crystal hydrodynamics:

$$
\begin{aligned}
\Pi_{\alpha \beta}^{\text {passive }}= & -P_{0} \delta_{\alpha \beta}+2 \xi\left(Q_{\alpha \beta}+\frac{1}{3} \delta_{\alpha \beta}\right) Q_{\gamma \epsilon} H_{\gamma \epsilon} \\
& -\xi H_{\alpha \gamma}\left(Q_{\gamma \beta}+\frac{1}{3} \delta_{\gamma \beta}\right)-\xi\left(Q_{\alpha \gamma}+\frac{1}{3} \delta_{\alpha \gamma}\right) H_{\gamma \beta} \\
& -\partial_{\alpha} Q_{\gamma \nu} \frac{\delta \mathcal{F}}{\delta \partial_{\beta} Q_{\gamma \nu}}+Q_{\alpha \gamma} H_{\gamma \beta}-H_{\alpha \gamma} Q_{\gamma \beta} .
\end{aligned}
$$

$P_{0}$ is a constant in the simulations reported here. The active term is given by $\prod_{\alpha \beta}^{\text {active }}=\zeta \Delta \mu Q_{\alpha \beta}$, where $\zeta$ is another 'activity' parameter. This equation is also valid at first order in $\Delta \mu[1]$.

The model above reduces to the Beris-Edwards model for liquid crystal hydrodynamics for $\lambda=\zeta=0$. For uniaxial active liquid crystals, the active liquid crystal director field $\vec{n}$ is well defined through $Q_{\alpha \beta}=3 q\left(n_{\alpha} n_{\beta}-\delta_{\alpha \beta} / 3\right) / 2$, where $q$ is the largest eigenvalue of $Q_{\alpha \beta}$. In this limit our model can be shown to reduce to the vectorial model considered in [1, 2].

We solve Eqs. 2 and 4 by using a Lattice Boltzmann algorithm. Our algorithm is a generalisation of the one introduced in Refs. [13] and successfully used to study a number of problems in 
the hydrodynamics and rheology of a passive liquid crystal. We now review it briefly, following the treatment in Ref. [13].

Lattice Boltzmann algorithms to solve the Navier-Stokes equations of a simple fluid, are defined in terms of a single set of partial distribution functions, the scalars $f_{i}(\vec{x})$, that sum on each lattice site $\vec{x}$ to give the density. For liquid crystal hydrodynamics, this must be supplemented by a second set, the symmetric traceless tensors $\mathbf{G}_{i}(\vec{x})$, that are related to the tensor order parameter $\mathbf{Q}$. Each $f_{i}, \mathbf{G}_{i}$ is associated with a lattice vector $\vec{e}_{i}[13]$. We choose a 15-velocity model on the cubic lattice with lattice vectors:

$$
\begin{aligned}
& \vec{e}_{i}^{(0)}=(0,0,0) \\
& \vec{e}_{i}^{(1)}=( \pm 1,0,0),(0, \pm 1,0),(0,0, \pm 1) \\
& \vec{e}_{i}^{(2)}=( \pm 1, \pm 1, \pm 1) .
\end{aligned}
$$

The indices, $i$, are ordered so that $i=0$ corresponds to $\vec{e}_{i}^{(0)}, i=1, \cdots 6$ correspond to the $\vec{e}_{i}^{(1)}$ set and $i=7,14$ to the $\vec{e}_{i}^{(2)}$ set.

Physical variables are defined as moments of the distribution function:

$$
\rho=\sum_{i} f_{i}, \quad \rho u_{\alpha}=\sum_{i} f_{i} e_{i \alpha}, \quad \mathbf{Q}=\sum_{i} \mathbf{G}_{i} .
$$

The distribution functions evolve in a time step $\Delta t$ according to

$$
\begin{aligned}
f_{i}\left(\vec{x}+\vec{e}_{i} \Delta t, t+\Delta t\right)-f_{i}(\vec{x}, t) & =\frac{\Delta t}{2}\left[\mathcal{C}_{f i}\left(\vec{x}, t,\left\{f_{i}\right\}\right)+\mathcal{C}_{f i}\left(\vec{x}+\vec{e}_{i} \Delta t, t+\Delta t,\left\{f_{i}^{*}\right\}\right)\right] \\
\mathbf{G}_{i}\left(\vec{x}+\vec{e}_{i} \Delta t, t+\Delta t\right)-\mathbf{G}_{i}(\vec{x}, t) & =\frac{\Delta t}{2}\left[\mathcal{C}_{\mathbf{G} i}\left(\vec{x}, t,\left\{\mathbf{G}_{i}\right\}\right)+\mathcal{C}_{\mathbf{G} i}\left(\vec{x}+\vec{e}_{i} \Delta t, t+\Delta t,\left\{\mathbf{G}_{i}^{*}\right\}\right)\right]
\end{aligned}
$$

This represents free streaming with velocity $\vec{e}_{i}$ followed by a collision step which allows the distribution to relax towards equilibrium. The $\mathcal{C}$ 's are collision operator (detailed below) while $f_{i}^{*}$ and $\mathbf{G}_{i}^{*}$ are first order approximations to $f_{i}\left(\vec{x}+\vec{e}_{i} \Delta t, t+\Delta t\right)$ and $\mathbf{G}_{i}\left(\vec{x}+\vec{e}_{i} \Delta t, t+\Delta t\right)$ respectively. They are obtained by using $\Delta t \mathcal{C}_{\mathbf{f} i}\left(\vec{x}, t,\left\{\mathbf{f}_{i}\right\}\right)$ on the right hand side of Eq. (10) and a similar substitution in Eq. (11). Discretizing in this way, which is similar to a predictor-corrector scheme, has the advantages that lattice viscosity terms are eliminated to second order and that the stability of the scheme is improved.

The collision operators are taken to have the form of a single relaxation time Boltzmann equation, together with a forcing term

$$
\begin{gathered}
\mathcal{C}_{f i}\left(\vec{x}, t,\left\{f_{i}\right\}\right)=-\frac{1}{\tau_{f}}\left(f_{i}(\vec{x}, t)-f_{i}^{e q}\left(\vec{x}, t,\left\{f_{i}\right\}\right)\right)+p_{i}\left(\vec{x}, t,\left\{f_{i}\right\}\right), \\
\mathcal{C}_{\mathbf{G} i}\left(\vec{x}, t,\left\{\mathbf{G}_{i}\right\}\right)=-\frac{1}{\tau_{\mathbf{G}}}\left(\mathbf{G}_{i}(\vec{x}, t)-\mathbf{G}_{i}^{e q}\left(\vec{x}, t,\left\{\mathbf{G}_{i}\right\}\right)\right)+\mathbf{M}_{i}\left(\vec{x}, t,\left\{\mathbf{G}_{i}\right\}\right) .
\end{gathered}
$$

The form of the equations of motion follow from the choice of the moments of the equilibrium distributions $f_{i}^{e q}$ and $\mathbf{G}_{i}^{e q}$ and the driving terms $p_{i}$ and $\mathbf{M}_{i}$. The distributions $f_{i}^{e q}$ are constrained by

$$
\sum_{i} f_{i}^{e q}=\rho, \quad \sum_{i} f_{i}^{e q} e_{i \alpha}=\rho u_{\alpha}, \quad \sum_{i} f_{i}^{e q} e_{i \alpha} e_{i \beta}=-\Pi_{\alpha \beta}^{\text {passive }}+\rho u_{\alpha} u_{\beta}
$$


where the zeroth and first moments are chosen to impose conservation of mass and momentum. The second moment of $f^{e q}$ controls the symmetric part of the stress tensor, whereas the moments of $p_{i}$

$$
\sum_{i} p_{i}=0, \quad \sum_{i} p_{i} e_{i \alpha}=\partial_{\beta} \tau_{\alpha \beta}, \quad \sum_{i} p_{i} e_{i \alpha} e_{i \beta}=0
$$

impose the antisymmetric part of the stress tensor, which we have called $\tau_{\alpha \beta}$. For the equilibrium of the order parameter distribution we choose

$$
\sum_{i} \mathbf{G}_{i}^{e q}=\mathbf{Q}, \quad \sum_{i} \mathbf{G}_{i}^{e q} e_{i \alpha}=\mathbf{Q} u_{\alpha}, \quad \sum_{i} \mathbf{G}_{i}^{e q} e_{i \alpha} e_{i \beta}=\mathbf{Q} u_{\alpha} u_{\beta} .
$$

This ensures that the order parameter is convected with the flow. Finally the evolution of the order parameter is most conveniently modeled by choosing

$$
\sum_{i} \mathbf{M}_{i}=\Gamma \mathbf{H}(\mathbf{Q})+\mathbf{S}(\mathbf{W}, \mathbf{Q}) \equiv \hat{\mathbf{H}}, \quad \sum_{i} \mathbf{M}_{i} e_{i \alpha}=\left(\sum_{i} \mathbf{M}_{i}\right) u_{\alpha}
$$

which ensures that the fluid minimises its free energy at equilibrium.

Conditions (14)-(17) are satisfied, by writing the equilibrium distribution functions and forcing terms as polynomial expansions in the velocity. The coefficients in the expansion are (in general

non-uniquely) determined by the requirements that these constraints are fulfilled (see Ref. [13] for details).

The active contributions then alter the constraints on the second moment of the $f_{i}$ 's and the first moment of the $\mathbf{G}_{i}$ 's distribution functions as follows:

$$
\sum_{i} \mathbf{M}_{i}=\hat{\mathbf{H}}+\lambda \Delta \mu \mathbf{Q}, \quad \sum_{i} f_{i}^{e q} e_{i \alpha} e_{i \beta}=-\Pi_{\alpha \beta}^{\mathrm{passive}}+\rho u_{\alpha} u_{\beta}-\zeta \Delta \mu Q_{\alpha \beta}
$$

Alternatively, we can input the derivative of the pressure tensor as a body force (as in Eq. 15). This scheme rigorously ensures no spurious velocity in equilibrium [13, 14] and has been employed in the following.

\section{RESULTS}

In this Section we report the results obtained with lattice Boltzmann simulations of the dynamic behaviour of a slab of active liquid crystal, sandwitched between two fixed plates at a mutual distance $L$ along the $y$ axis (see Fig. 1 for axis definition). As a result our system is effectively one dimensional (the velocity and the active liquid crystal director fields depend on the velocity gradient coordinate, $y$, only) We shall consider different kinds of boundary conditions, and demonstrate that these affect the hydrodynamics of the fluid considerably.

The model parameters in the simulations reported here were typically fixed as follows: $A_{0}=$ $0.1, L=100, K=0.04, \gamma=3, \xi=0.7, \Gamma \sim 0.34$, while the relaxation time of the lattice Boltzmann evolution of the density distribution function (see Ref. [13] for details) is 0.7. All 
preceding values are in simulation units. These can be straightforwardly related to a set of physical units by considering that one space and time simulation units correspond to $0.02 \mu \mathrm{m}$ and 0.067 $\mu$ s respectively (for how to do this mapping see Ref. [13]). One can thus map our sample onto a physical slab of active liquid crystal of thickness $10 \mu \mathrm{m}$, while the parameters of the liquid crystalline fluids are $K=16.2 \mathrm{pN}, \gamma=1$ Poise ( $\gamma$ is the rotational viscosity [15]), while the ratio between the Leslie viscosities $\alpha_{3} / \alpha_{2} \sim 0.08$. Note that a positive value of $\alpha_{3} / \alpha_{2}$ means that the liquid crystal in the passive phase is flow aligning [15]; thus the parameter choice made here corresponds to a flow aligning liquid crystal in the passive $(\zeta=0)$ phase. We have further fixed $\lambda=0$ in our simulations - as this parameter has been shown to not affect the existence of a transition between a passive quiescent and an active phase with spontaneous flow [2]. The values of the remaining 'activity' parameter $\zeta$ were on the other hand systematically varied.

In what follows the director field (also called polarisation field in the context of active gels in Ref. [2]) remains in the $x y$ plane in the vast majority of cases. It is therefore useful to introduce an orientation angle $\theta$, as the angle between the director field and the positive direction along the $y$ axis.

Note that, unless specified otherwise, we always initialised the system with zero velocity field and with a director field corresponding to the stable equilibrium one in the passive phase. However, as this initial state is generally metastable, and as our lattice Boltzmann simulations do not include noise, we introduced a small perturbation - typically in the form of a small deviation in the orientation angle at $y=L / 2$. This is enough to allow the system to escape from the metastable passive phase in which it is initialised.

\section{Active liquid crystals with normal anchoring}

We first consider an active liquid crystal with normal anchoring at its boundaries (Fig. 1a).

For values of $\zeta$ smaller than $\zeta_{c} \sim 0.0015$ the liquid crystal remains in the passive phase, whereas for $\zeta>\zeta_{c}$ there is a finite flow along the $x$ direction (Fig. 1), i.e. the liquid crystal is in an active phase. Fig. 2 shows the director and velocity profiles first encountered in the active phase upon increasing the value of $\zeta$. There is a net primary flow along the $x$ direction, which exhibits a sharp maximum at the channel center, and is quite different from e.g. the common parabolic Poiseuille velocity profile which may be externally induced in a passive liquid crystal by exerting a pressure difference or a body force on it. The spontaneous flow symmetrically deforms the director profile and two 'bands' develop, in which the orientation angle $\theta$ attains opposite values (close to that of the Leslie angle [15] which is imposed by the local shear rate introduced by the spontaneous flow). These band flatten when the value of $\zeta$ is increased - up to a second critical value, which for the parameters we use here is $\sim 0.0015$.

A further increase in $\zeta$ leads to a qualitatively different behaviour, as shown in Fig. 3. The velocity profile changes and now has a sinusoidal shape, so that it is zero at the centre of the channel and there is no net flow. For values of $\zeta$ close to this second crossover the director field 
exhibits an out-of-plane instability, much as conventional (yet flow tumbling) liquid crystals under strong shear. For large $\zeta$ the director field is well aligned along the shear direction (Fig. 3).

\section{Active liquid crystal with conflicting anchoring}

We now come to the case of an active liquid crystal with the director field along $y$ at $y=L$ and along $x$ at $y=0$. In the passive case the liquid crystal responds to this conflicting anchoring by adopting a uniform (in the one elastic constant approximation employed here) splay-bend deformation fully contained in the $x y$ plane.

Fig. 4 shows the director and velocity fields at steady state for small values of the activity $\zeta$. Contrarily to the normal anchoring case, we find no critical non-zero value of $\zeta$ for which the system is passive. Formally this can be understood as in the passive steady state there are gradients of $\mathrm{Q}$ which set up a non-trivial shear rate so that the passive solution is always unstable (for $\zeta>0$ ). For small values of $\zeta$, the active gel expands the region which is aligned along $x$, while the flow field is asymmetric at steady state.

Fig. 5 proves that also in this HAN geometry further 'transitions' are encountered when going deeper into the active phase by increasing $\zeta$. For $\zeta>\zeta^{*} \sim 0.0003$, the sign of the flow in steady state reverses. Increasing the activity also leads to a more symmetric, Poiseuille-like flow. The change in orientation of the fluid flow relative to the director field leads to a strikingly different elastic deformation which ends up with a point with $\theta=0$ (director completely along the velocity gradient direction) within the sample. The director profile can be understood as due to the fluid flow in steady state, as it resembles the elastic distortions in steady state found with clockwise

and anti-clockwise HAN cells under a constant shear flow in Ref. [18]. It would be of interest to develop a semi-analytic theory which accounts for the presence of extra 'transitions', both in the HAN and in the normal anchoring geometry, when the activity is increased.

\section{Dynamic pathway to steady state}

We now consider the dynamic pathway followed by the active liquid crystal to reach its steady state. For concreteness, henceforth we will restrict to the case of normal anchoring.

Fig. 6 show the dynamics followed by a system with $L=40 \mu \mathrm{m}$, which started from a (slightly perturbed) spatially constant director field with normal anchoring and no flow. The value of the activity parameter is one for which, for $L=10 \mu \mathrm{m}$, one ends up with states similar to those in Fig. 2. In the larger sample this value of $\zeta$ is enough to lead to an out-of-plane director field in steady state. This is in line with previous observations that larger systems are more easily led into an active state [2]. The early time dynamics is characterised by the generation of a lot of deformation in the director field, (reflected in the large spatial changes in $n_{x, y, z}$ ), caused by the appearance of spontaneous flow. Thermodynamic forces then set in to reduce the number of 'bands' or equivalently to reduce the amount of splay and bend. Note also that transiently the 
director field develops a significant asymmetry, so that it is twisted unevenly, which gradually disappears upon approaching the steady state.

Note that we have also simulated situations in which the activity value is cyclically increased and decreased so that the initial condition is different in the two halves of a 'cycle', to compare the steady states found in the two cases (data not shown). While with small to moderate values of the activity, for which the active phase has just set in, we find no dependence on the initial conditions, this is not true as we go deeper in the active phase. In this region we find there is some hysteresis, and that the steady state does depend on initial condition for large value of $\zeta$ (i.e. deep in the active phase). This is perhaps not surprising because when subjected to a flow (in this case stimulated by the activity), liquid crystals and other viscoelastic gels may exhibit rheochaotic (hence strongly initial condition dependent) flow properties [17]. It would be interesting to systematically characterise the dependence of steady state and of the dynamic pathway in active gels for large $\zeta$, and determine whether, under appropriate conditions, complex, or even chaotic, dynamics are observed. This is deferred to future work.

\section{CONCLUSIONS AND DISCUSSIONS}

In conclusions, we have presented a generalised lattice Boltzmann algorithm, inspired by those previously used to solve the Beris-Edwards equations of motion of a passive liquid crystal, to investigate numerically the hydrodynamic properties of an active (flow-aligning) liquid crystalline fluid in an effectively 1D geometry. We have studied two distinct boundary conditions (one with the director field normally anchored at the boundary plates and another one with conflicting anchoring leading to elastic distortions even in the passive phase), and systematically considered how the director and flow fields in steady state evolve upon increasing the parameter $\zeta$, which is a measure of the liquid crystal 'activity'.

The interplay of activity, which may introduce spontaneous flow in steady state, and the elastic distortion caused by the fixed anchoring at the boundaries, gives rise to an interesting phenomenology. We show that our algorithm gives a non-equilibrium transition from a passive to an active phase, and that this occurs with zero threshold when there is conflicting anchoring at the boundaries. Deep in the active phase, where semi-analytical methods are typically unhelpful, we find further 'transitions' between qualitatively different steady states of flow and director field. When normal anchoring is considered, we observe banded flow solutions in which regions of shearoriented filaments or director field are separated by regions of bend-splay distortions (where the order parameter decreases slightly), as well as states with director field out of the $x z$ plane in steady state.

The equations of motion we have studied are expected to describe the coarse grained dynamics of actomyosin solutions or of microtubular networks with dyneins, when the actin fibers or the microtubules have a fixed orientation at the surface (due e.g. to chemical or mechanical treatment of the surface as in the csae of passive liquid crystal). We note that our algorithm can be 
straightforwardly applied to 2D and 3D problems; this will be pursued elsewhere.

[1] K. Kruse, J. F. Joanny, F. Julicher, J. Prost, K. Sekimoto, Eur. Phys. J. E 16, 5 (2005); Phys. Rev. Lett. 92, 078101 (2004).

[2] R. Voituriez, J. F. Joanny, J. Prost, Europhys. Lett. 70, 404 (2005); Phys. Rev. Lett. 96, 028102 (2006).

[3] R. A. Simha, S. Ramaswamy, Phys. Rev. Lett. 89, 058101 (2002).

[4] Y. Hatwalne, S. Ramaswamy, M. Rao, R. A. Simha, Phys. Rev. Lett. 92, 118101 (2004).

[5] T. B. Liverpool, M. C. Marchetti, Phys. Rev. Lett. 90, 138102 (2001); Europhys. Lett. 69, 846 (2005).

[6] D. Bray, Cell movements: from molecules to motility, Garland Publishing, New York (2000).

[7] J. Howard, Mechanics of Motor Proteins and the Cytoskeleton, Sinauer Associates, Inc., Sunderland (2001).

[8] J. van der Gucht, E. Paluch, C. Sykes, Proc. Natl. Acad. Sci. USA 102, 7847 (2005).

[9] P. R. Cook, Principles of Nuclear Structure and Function, Wiley, New York (2001).

[10] D. Humphrey, C. Duggan, D. Saha, D. Smith, J. Kas,Nature 416, 413 (2002).

[11] F. Nedelec, T. Surrey, E. Karsenti, Curr.Opin. Cell Biol. 15, 118 (2003).

[12] C. Storm, J. J. Pastore, F. C. MacKintosh, T. C. Lubensky, P. A. Janmey, Nature 435, 191 (2005).

[13] C. Denniston, D. Marenduzzo, E. Orlandini, J. M. Yeomans, Phil. Trans. Roy. Soc. A 362, 1745 (2004); C. Denniston, E. Orlandini, Y. M. Yeomans, Phys. Rev. E 63, 056702 (2001); D. Marenduzzo, E. Orlandini, J. M. Yeomans, Phys. Rev. Lett. 92, 188301 (2004); C. M. Care, D. J. Cleaver, Rep. Prog. Phys. 68, 2665 (2005).

[14] A. J. Wagner, Int. J. Mod. Phys. B 17, 193 (2003).

[15] P.G. de Gennes J. Prost, The Physics of Liquid Crystals, 2nd Ed., Clarendon Press, Oxford, (1993).

[16] A.N. Beris, B.J. Edwards, Thermodynamics of Flowing Systems, Oxford University Press, Oxford, (1994).

[17] B. Chakrabarti, M. Das, C. Dasgupta, S. Ramaswamy, A. K. Sood, Phys. Rev. Lett. 92, 055501 (2004); A. Aradian, M. E. Cates, Europhys. Lett. 70, 397 (2005); S.M. Fielding, P.D. Olmsted, Phys. Rev. Lett. 92, 084592 (2004).

[18] D. Marenduzzo, E. Orlandini, J. M. Yeomans, Europhys. Lett. 64, 406 (2003); J. Chem. Phys. 121, 582 (2004). 


\section{Figure captions}

Fig. 1: Geometry used for the calculations described in the text. The active gel is sandwiched between two infinite plates, parallel to the $x z$ plane, lying at $y=0$ and $y=L$. We consider (a) normal anchoring and (b) conflicting anchoring (leading to a hybrid aligned nematic cell for a passive liquid crystal)

Fig. 2: Director (left) and velocity (right) fields in steady state for an active liquid crystal slab with normal anchoring at the boundaries, and $\zeta$ equal to 0.0002 (solid line), 0.0004 (longdashed line), 0.0006 (dot-dashed line), and 0.001 (dotted line), respectively. Other parameters are as specified in the text.

Fig. 3: Director (left) and velocity (right) fields in steady state for an active liquid crystal slab with normal anchoring at the boundaries, and $\zeta$ equal to 0.002 (solid line), 0.003 (longdashed line), 0.005 (dot-dashed line), and 0.007 (dotted line) respectively. Other parameters are as specified in the text.

Fig. 4: Director (left) and velocity (right) fields in steady state for an active liquid crystal slab with conflicting anchoring at the boundaries (Fig. 1, and $\zeta$ equal to 0.00001 (solid line), 0.00005 (long-dashed line), 0.0001 (dot-dashed line), and 0.0002 (dotted line), respectively. Other parameters are as specified in the text.

Fig. 5: Director (left) and velocity (right) fields in steady state for an active liquid crystal slab with conflicting anchoring at the boundaries (Fig. 11 and $\zeta$ equal to 0.0004 (solid line), 0.0006 (long-dashed line), 0.0008 (dot-dashed line), and 0.003 (dotted line), respectively. Other parameters are as specified in the text.

Fig. 6: Dynamic pathway to steady state followed by a system with $\zeta=0.0008, L=400$, and other parameters as in the previous section. Solid, long dashed and dot-dashed lines correspond to the $z, x$ and $y$ components of the director field respectively. 
(a)

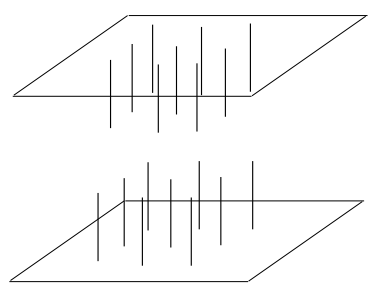

(b)

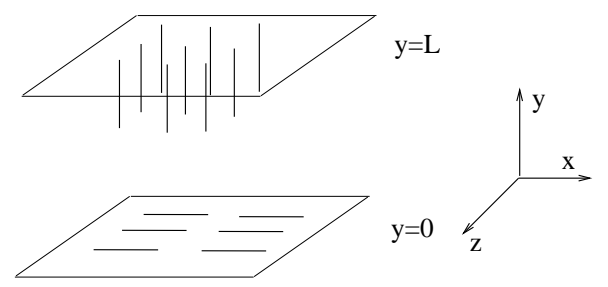

FIG. 1: Geometry used for the calculations described in the text. The active gel is sandwiched between two infinite plates, parallel to the $x z$ plane, lying at $y=0$ and $y=L$. We consider (a) normal anchoring and (b) conflicting anchoring (leading to a hybrid aligned nematic cell for a passive liquid crystal). 

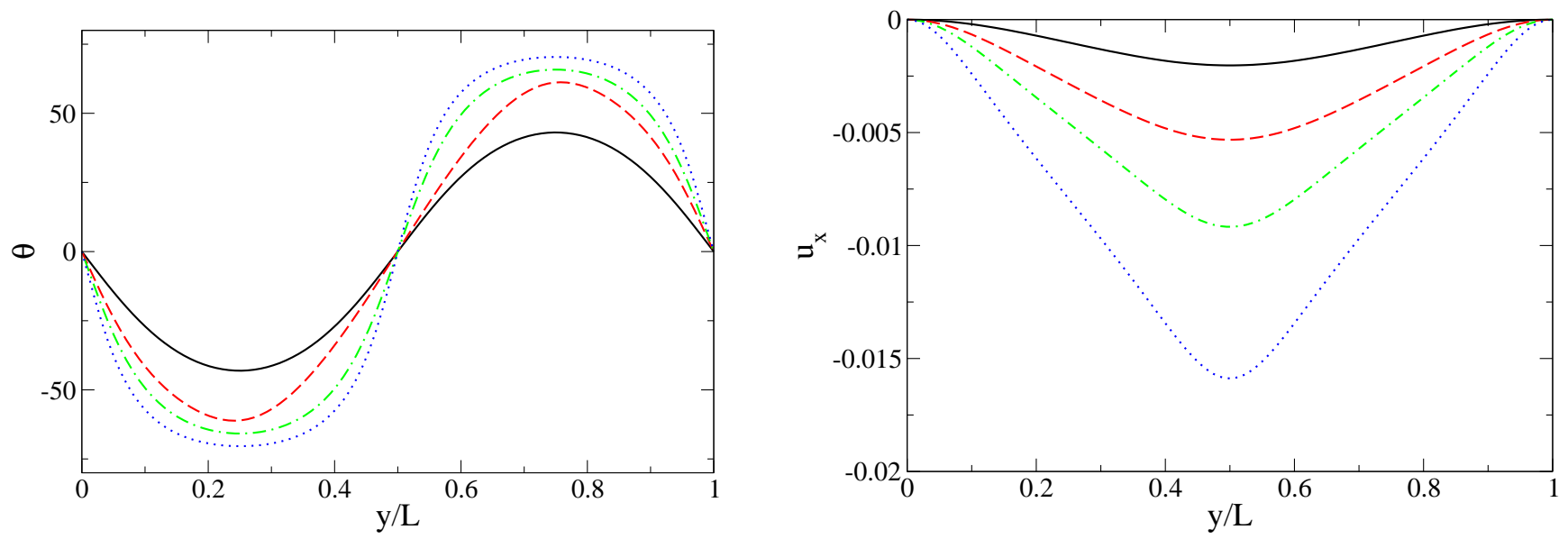

FIG. 2: Director (left) and velocity (right) fields in steady state for an active liquid crystal slab with normal anchoring at the boundaries, and $\zeta$ equal to 0.0002 (solid line), 0.0004 (long-dashed line), 0.0006 (dot-dashed line), and 0.001 (dotted line), respectively. Other parameters are as specified in the text. 

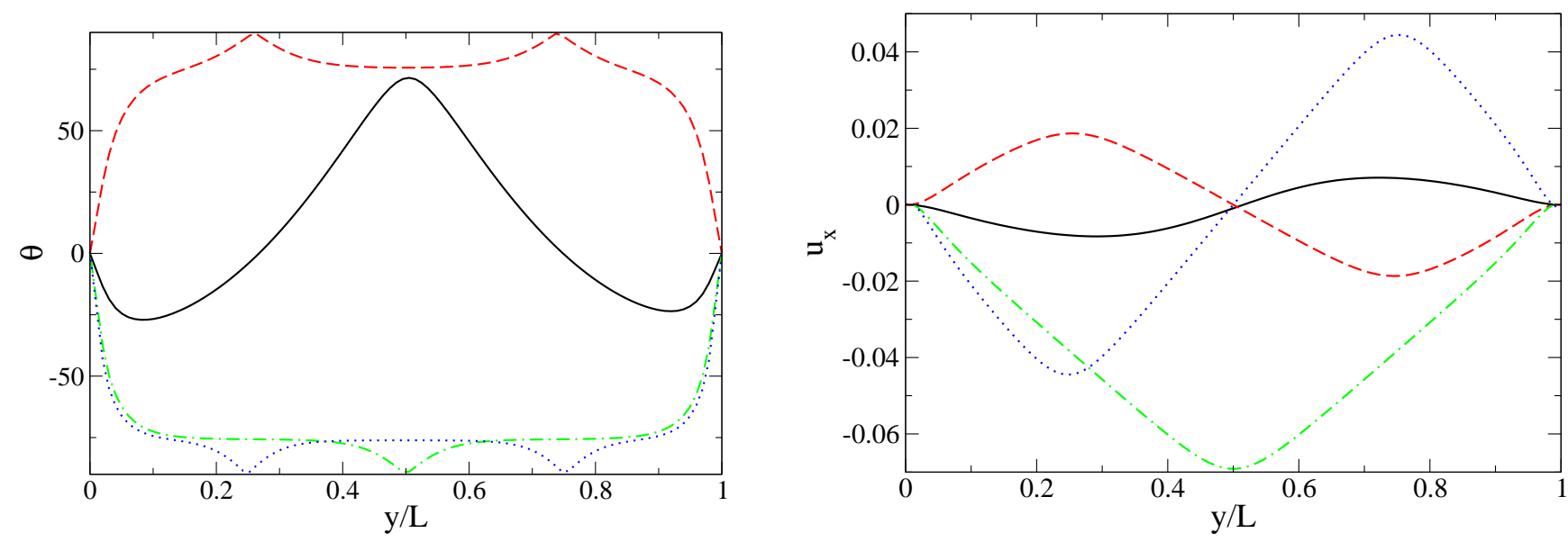

FIG. 3: Director (left) and velocity (right) fields in steady state for an active liquid crystal slab with normal anchoring at the boundaries, and $\zeta$ equal to 0.002 (solid line), 0.003 (long-dashed line), 0.005 (dot-dashed line), and 0.007 (dotted line) respectively. Other parameters are as specified in the text. 

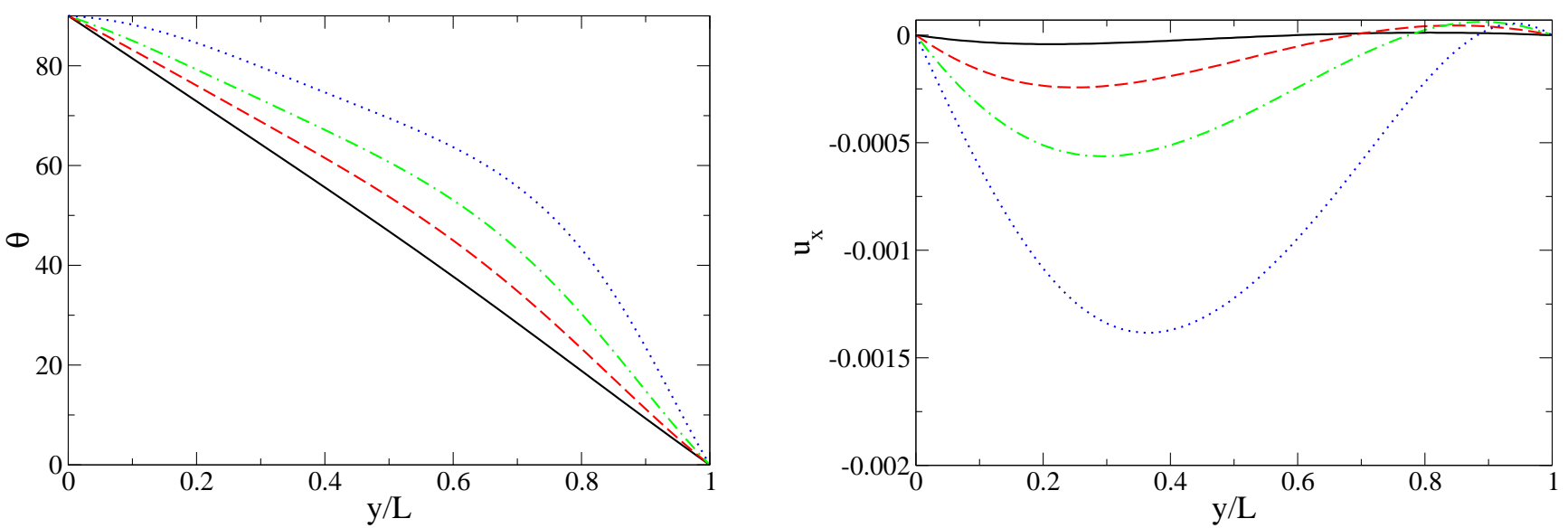

FIG. 4: Director (left) and velocity (right) fields in steady state for an active liquid crystal slab with conflicting anchoring at the boundaries (Fig. 1, and $\zeta$ equal to 0.00001 (solid line), 0.00005 (long-dashed line), 0.0001 (dot-dashed line), and 0.0002 (dotted line), respectively. Other parameters are as specified in the text. 

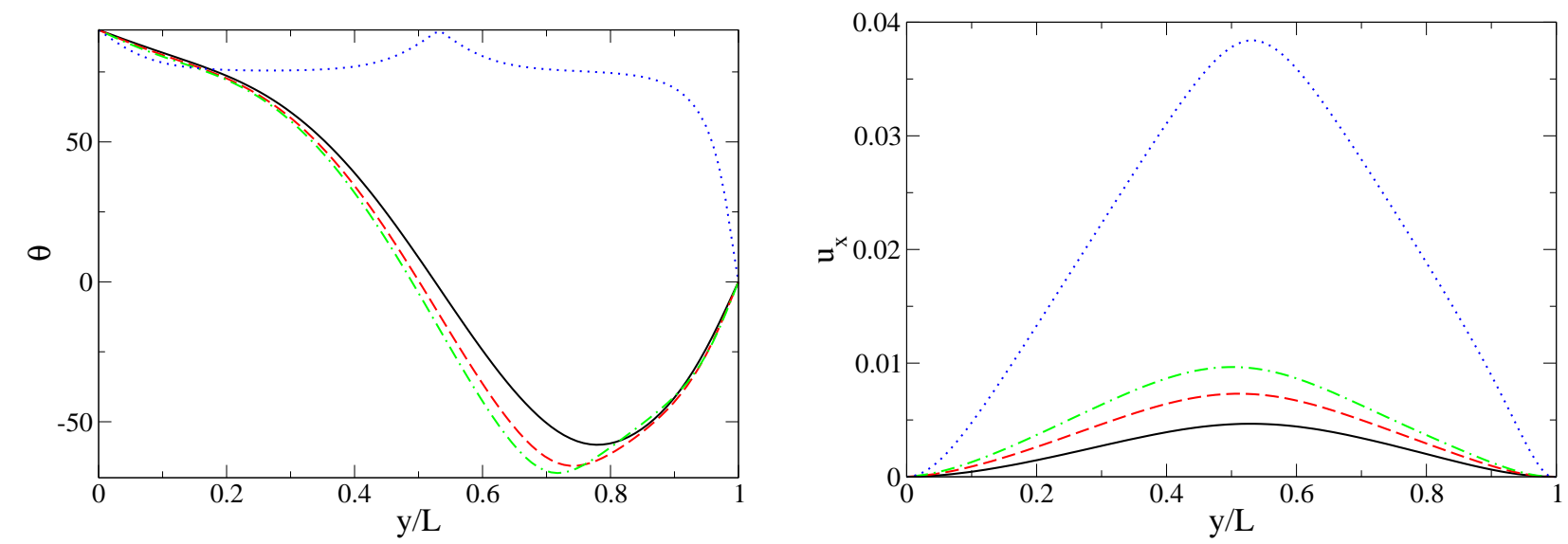

FIG. 5: Director (left) and velocity (right) fields in steady state for an active liquid crystal slab with conflicting anchoring at the boundaries (Fig. 1) and $\zeta$ equal to 0.0004 (solid line), 0.0006 (long-dashed line), 0.0008 (dot-dashed line), and 0.003 (dotted line), respectively. Other parameters are as specified in the text. 

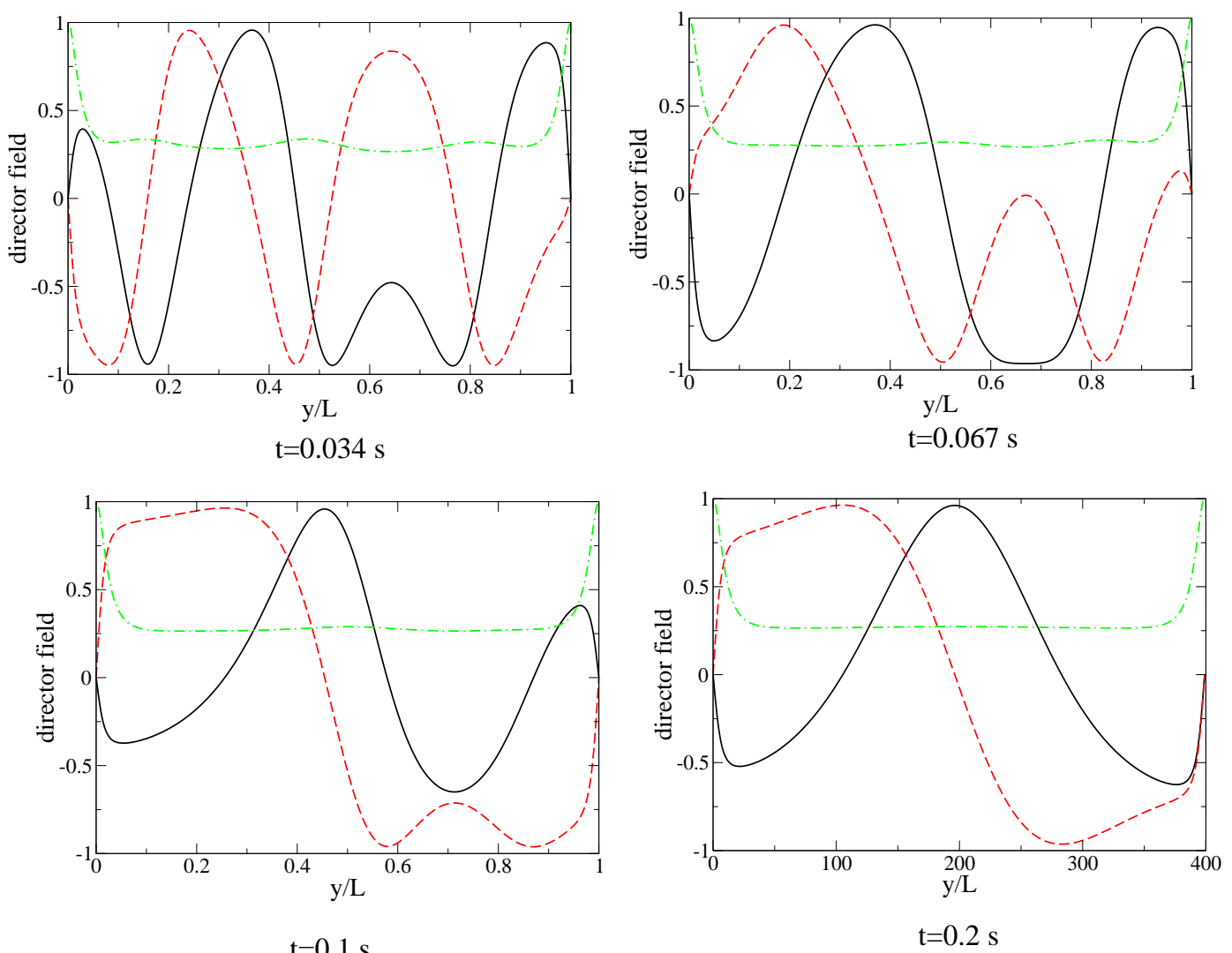

FIG. 6: Dynamic pathway to steady state followed by a system with $\zeta=0.0008, L=400$, and other parameters as in the previous section.Solid, long dashed and dot-dashed lines correspond to the $z, x$ and $y$ components of the director field respectively. 